\title{
Enquête séroépidémiologique de la rhinopneumonie des équidés en Tunisie
}

\author{
A. Ghram ${ }^{1}$ A. Chabchoub ${ }^{2 *}$ M. Boussetta ${ }^{1}$ \\ S. Baazaoui ${ }^{3}$ H. Ibn Amor ${ }^{3}$ F. Landolsi ${ }^{2}$
}

\section{Mots-clés}

Cheval Pur-sang Arabe - Cheval Pursang Anglais - Cheval Barbe - Cheval Arabe-Barbe - Virus rhinopneumonie équine - Réaction de fixation du complément - Immunologie - Epidémiologie - Tunisie.

\begin{abstract}
Résumé
Une enquête séroépidémiologique, réalisée sur 789 équidés (400 élevés au Nord-Est de la Tunisie, 389 dans la région du Sahel et du Centre), a permis de détecter, par le test de fixation du complément, des anticorps spécifiques contre le virus de la rhinopneumonie équine. Les résultats ont montré que 15 équidés $(1,9$ p. 100) étaient séropositifs, avec des taux variables d'anticorps fixant le complément. Ces résultats sont discutés en relation avec ceux obtenus par d'autres auteurs en Tunisie et dans les pays voisins.
\end{abstract}

Les équidés, en particulier le cheval, ont un rôle socio-économique important en Tunisie. Cependant, l'état de santé du cheptel équin reste toujours menacé par la survenue de certaines maladies infectieuses et contagieuses qui entraînent une morbidité et parfois une mortalité dans les élevages infectés. La rhinopneumonie des équidés, sous sa forme respiratoire, abortive ou nerveuse, est une pathologie parfois redoutée. Elle engendre des pertes économiques importantes, immobilise les effectifs séropositifs et perturbe les activités équestres.

Peu d'informations sont disponibles sur la situation épidémiologique de cette maladie en Tunisie. Les auteurs ont jugé utile de mener une enquête séroépidémiologique dans le Nord puis dans le Centre du pays pour mieux évaluer la prévalence de cette entité pathologique.

1. Laboratoire de microbiologie vétérinaire, Institut Pasteur de Tunis, BP 74, 13 place Pasteur, 1002 Tunis Belvédère, Tunisie

2. Service de pathologie médicale des équidés et carnivores, Ecole nationale de médecine vétérinaire, 2020 Sidi-Thabet, Tunisie

Tél. : 002161552200 ; Fax : 002161552441

3. Circonscription de la Production animale, 3130 Haffouz, Gouvernorat de Kairouan, Tunisie

* Auteur pour la correspondance
MATERIEL ET METHODES

\section{Région}

L'enquête s'est portée sur un effectif d'équidés dans des régions où le cheptel équin, en particulier de race pure, est assez important. Il s'agit des gouvernorats du Nord (Tunis, Ariana et Bizerte), ainsi que du Sahel et du Centre (Sousse, Monastir, Mahdia, Kairouan et Sidi Bouzid).

\section{Animaux}

Un effectif total de 789 équidés a été étudié. Des chevaux Pursang Arabes (PsAr), stationnés à la FNARC (Fondation nationale d'amélioration de la race chevaline) à Sidi-Thabet (Ariana), des Pur-sang Anglais (PsAng) d'un haras privé au gouvernorat de Bizerte et d'un effectif de chevaux Barbes (Ba) et Arabes-Barbes ( $\mathrm{ABa}$ ) élevés à la Mannouba (Ariana) ont constitué le total des équidés prélevés dans le Nord.

De même, 389 équidés de différentes races provenaient des régions du Sahel et du Centre tunisien. La répartition de tout l'effectif étudié selon l'espèce et la race figure dans le tableau I.

\section{Prélèvement}

Une seule prise de sang a été effectuée à la veine jugulaire des animaux étudiés. Le sérum a ensuite été séparé et conservé-congelé à $-20{ }^{\circ} \mathrm{C}$ jusqu'à l'analyse par la technique de fixation du complément (FC). 


\section{Tableau I}

Taux d'infection observé selon la race et l'espèce des équidés explorés

\begin{tabular}{|c|c|c|c|}
\hline Race/espèce & $\begin{array}{l}\mathrm{Nb} \text {. d'animaux } \\
\text { explorés }\end{array}$ & $\begin{array}{l}\text { Sérums } \\
\text { positifs }\end{array}$ & $\begin{array}{l}\text { Taux d'in- } \\
\text { fection (\%) }\end{array}$ \\
\hline Pur-sang Arabe & 373 & 5 & 1,66 \\
\hline Pur-sang Anglais & 8 & 0 & 0 \\
\hline Barbe & 20 & 0 & 0 \\
\hline Arabe-Barbe & 80 & 0 & 0 \\
\hline Poney et Breton & 6 & 0 & 0 \\
\hline $\begin{array}{l}\text { Chevaux d'origine } \\
\text { inconnue }\end{array}$ & 83 & 6 & 7,22 \\
\hline Anes et mulets & 154 & 4 & 2,60 \\
\hline Total & 789 & 15 & 1,90 \\
\hline
\end{tabular}

\section{Méthodes}

\section{Préparation des antigènes}

L'antigène, une suspension du virus de la rhinopneumonie (equine herpes virus-1 (EHV1)), aimablement fourni par le laboratoire central (CNEVA), Maisons-Alfort, France, a été préparé sur des cultures de lignée cellulaire Vero. Des cultures de cellules en monocouche ont été infectées et incubées à $37^{\circ} \mathrm{C}$ pendant 4 à $5 \mathrm{j}$. Lorsque l'effet cytopathique a atteint 80 p. 100 du tapis cellulaire, les cultures ont été collectées, congelées-décongelées deux fois, puis centrifugées à $3000 \mathrm{t} / \mathrm{min}$ pendant $30 \mathrm{~min}$ pour éliminer les débris cellulaires. Le surnageant a été collecté, réparti en aliquotes de $1 \mathrm{ml}$ et congelé à $-80^{\circ} \mathrm{C}$.

\section{Titrage des antigènes}

Le stock viral, prétitré sur cultures de cellules Vero, constitue l'antigène viral utilisé dans la technique de fixation du complément. Pour déterminer la dilution de l'antigène nécessaire pour l'analyse sérologique des sérums prélevés, un titrage en échiquier a été réalisé, opposant des dilutions en série d'un sérum positif de titre connu (1/160) à des dilutions en série de l'antigène (titrant au moins $10^{5}$ DICC50), en présence de 2 UI de complément de cobaye. La plus forte dilution de l'antigène (dilution au 1/4) donnant le titre le plus élevé avec le sérum positif (inhibition totale de l'hémolyse) correspondait à une unité antigénique.

\section{Titrage des sérums}

Pour la mise en évidence des anticorps fixant le complément dans les sérums équins collectés, la technique LBCF (laboratory branch complement fixation), adaptée en microméthode sur plaque (3), a été utilisée.

Les sérums ont été décomplémentés à $56{ }^{\circ} \mathrm{C}$ pendant $30 \mathrm{~min}$. Ils ont été dilués extemporanément selon une progression géométrique de raison 2 à partir de la dilution initiale de $1 / 8$, et placés à raison de $25 \mu \mathrm{l}$ par puits d'une plaque à 96 puits. L'antigène, dilué au 1/4 dans un tampon Veronal, $\mathrm{pH} 7,3$, de façon à contenir une unité antigénique dans un volume de $25 \mu \mathrm{l}$, a été placé dans chaque puits et la plaque a été incubée $10 \mathrm{~min}$ à la température ambiante. Le complément, prétitré avant chaque série de réaction, a été ajouté à raison de 2 UI par $50 \mu$ let par puits et la plaque placée à $37^{\circ} \mathrm{C}$ pendant $30 \mathrm{~min}$.
Le système hémolytique utilisait des hématies de mouton à 2 p. 100 dans un tampon Veronal, $\mathrm{pH}$ 7,3. Le sérum hémolytique est un sérum anti-hématies de mouton préparé chez le lapin et utilisé à la dilution 1/500. La sensibilisation des hématies est réalisée au moment de l'emploi.

La lecture de la plaque se fait à 100 p. 100 d'inhibition de l'hémolyse ; un sérum est considéré positif s'il présente un titre de 8 ou plus.

\section{- RESU LTATS}

Le tableau I présente les résultats sérologiques obtenus par la technique de FC chez les différentes espèces et races d'équidés testés. Parmi les 789 sérums analysés, 15 se sont révélés positifs avec des titres sériques supérieurs ou égaux à 16. Un taux d'infection global de 1,9 p. 100 a été observé.

Les résultats montrent que seuls les PsAr, les chevaux d'origine inconnue (OI), les ânes et les mulets présentaient des taux significatifs d'anticorps antivirus de la rhinopneumonie (RH), les OI étant les plus touchés par cette infection avec un taux d'infection de 7,22 p. 100.

Par contre, une sérologie négative a été observée chez les PsAng, les $\mathrm{Ba}$ et les poneys. Le nombre d'animaux testés était cependant trop réduit pour pouvoir interpréter les résultats.

Les titres sériques observés variaient entre 16 et 256 , avec 7 sérums ayant des titres de 16, 7 des titres de 32 et 1 des titres de 256 .

Les équidés ayant une sérologie positive ont été retrouvés aussi bien au Nord (1,25 p. 100) qu'au Sahel (1,6 p. 100) et au Centre (4,12 p. 100), les équidés du gouvernorat de Kairouan (haras) montrant le taux d'infection le plus élevé.

Le taux de séropositivité le plus élevé $(7,8$ p. 100) a été observé chez les équidés âgés entre 0 et 2 ans. Au fur et à mesure que la tranche d'âge augmentait, le taux d'infection diminuait, atteignant 0,8 p. 100 chez les chevaux âgés de plus de 10 ans (tableau II). La différence entre les taux d'infection des tranches d'âge considérées est significative $(\mathrm{p}<0,05)$.

Considérant le sexe des équidés examinés, il s'avère que le taux d'infection chez les mâles séropositifs est similaire à celui des femelles et se situe à 1,9 p. 100 .

\section{DISCUSSION}

Cette enquête a révélé, chez les 789 équidés prélevés, un taux de séroprévalence pour la rhinopneumonie de 1,9 p. 100. Ce taux peut être considéré comme faible si on le compare à ceux rapportés au Maroc (45 p. 100) (9) et en France (30 p. 100) (10).

Les résultats de cette étude peuvent être rapprochés de ceux obtenus en Tunisie en 1980 par Ellouze (5) qui a rapporté un taux d'infection de 6 p. 100. Par contre, les études menées par Mérai (8) en 1985 et Toumi (11) en 1986 n'ont révélé aucune trace sérologique de la rhinopneumonie par la technique de fixation du complément. Néanmoins, la séroneutralisation appliquée aux mêmes sérums avait permis de détecter au moins deux juments séropositives au $1 / 32$. Ceci pouvait témoigner d'une infection ancienne $(4,12)$.

Il apparaît que les chevaux d'origine inconnue, les mulets et les ânes étaient les plus atteints par cette infection. Cependant, il est important de remarquer que l'infection a aussi semblé affecter les PsAr qui ont présenté un taux d'infection de 1,66 p. 100. 
Tableau II

Nombre et pourcentage d'équidés séropositifs envers la rhinopneumonie en fonction des tranches d'âge considérées

\begin{tabular}{|c|c|c|c|c|c|c|c|}
\hline \multirow{2}{*}{$\begin{array}{l}\text { Animaux } \\
\text { explorés }\end{array}$} & \multicolumn{6}{|c|}{ Tranches d'âge considérées } & \multirow[t]{2}{*}{ Total } \\
\hline & 0 à 2 ans & 2 à 4 ans & 4 à 6 ans & 6 à 8 ans & 8 à 10 ans & $>10$ ans & \\
\hline Nombre d'équidés & 64 & 61 & 122 & 93 & 80 & 369 & 789 \\
\hline Nombre de positifs & 5 & 2 & 3 & 1 & 1 & 3 & 15 \\
\hline$\%$ positifs & 7,58 & 3,2 & 2,45 & 1 & 1,25 & 0,8 & 1,9 \\
\hline
\end{tabular}

En fait, l'infection a été retrouvée dans les régions du Nord, du Sahel ou du Centre du pays avec des taux variables. Ceci montre le potentiel de diffusion du virus de la RH parmi le cheptel équin. Par contre, sa persistance serait probablement liée aux conditions d'entretien des équidés. En effet, les chevaux appartenant à des élevages organisés ont présenté les taux d'infection les plus faibles (exemple du haras privé du gouvernorat de Bizerte).

Aucune différence significative n'a pu être démontrée entre la séropositivité des mâles et celle des femelles. Ceci est compatible avec ce qui a été rapporté par ailleurs $(4,6,9,11)$. Par contre, l'âge a semblé jouer un rôle dans la transmission et la persistance du virus de la RH. Ainsi, les jeunes animaux ont présenté le taux d'infection le plus élevé et ce taux a eu tendance à décroître avec l'âge. Ceci rejoint les constatations de Moraillon et coll. (9) qui rapportent une séropositivité plus importante chez les jeunes que chez les adultes.

La détection de taux d'anticorps anti-RH chez les jeunes pourrait être liée à la prise colostrale et au mode de contagion des herpès virus qui se transmettent essentiellement par la voie respiratoire et au jeune âge. En effet, Delannoy et coll. (4) ainsi que Zientra et coll. (13) rapportent que la majorité des jeunes chevaux sont en contact, au cours de leur première année de vie, avec l'EHV1 et l'EHV2, responsables d'épizooties essentiellement observées en automne et en hiver. Par contre, chez l'adulte ce sont surtout les formes subcliniques ou parfois inapparentes qui sont les plus fréquentes.

Chez le poulain, une atteinte de l'ensemble des alvéoles pulmonaires suivie d'une broncho-pneumonie a été constatée. Alors que l'EHV4 ne semble pas dépasser ce stade local, l'EHV1 passe rapidement dans le torrent circulatoire par le biais des leucocytes qu'il infecte, contaminant ainsi d'autres organes cibles (4).

Enfin, les anticorps fixant le complément sont les premiers à apparaître et disparaissent rapidement après l'infection. Les taux d'anticorps anti-RH, révélés par la technique $\mathrm{FC}$, indiquent une infection récente, ce qui plaide en faveur d'une circulation « à bas bruit » de cette infection dans les élevages équins tunisiens.

\section{CON CLUSION}

Les résultats de cette enquête séroépidémiologique ont permis de montrer l'existence et la persistance du virus de la rhinopneumonie dans les élevages des équidés en Tunisie. Cette entité pathologique mérite ainsi toute l'attention des éleveurs et des responsables des élevages équins. Il serait judicieux de la rechercher systématiquement lors de troubles respiratoires et surtout lors d'avortement en réalisant des prélèvements d'écouvillons nasaux ou vaginaux et de l'avorton pour la recherche du virus responsable.
De même, le suivi du statut immunologique des chevaux est d'autant plus important que la vaccination contre la rhinopneumonie n'est pas systématiquement appliquée dans le pays. La prophylaxie sanitaire reste ainsi le seul moyen à utiliser pour lutter contre cette pathologie grave.

Il conviendrait également de continuer à faire des sondages sérologiques dans les différents haras de Tunisie en utilisant, en plus de la FC qui détecte une infection récente, la technique de séroneutralisation qui révèle les anticorps neutralisants plus tardifs et persistants, permettant une meilleure appréciation de la prévalence de la rhinopneumonie en Tunisie.

\section{Remerciements}

Les auteurs remercient le Secrétariat d'Etat à la Recherche scientifique et technique (SERST) pour son aide matérielle qui a permis le financement d'une partie de ces travaux. De même, ils remercient les responsables de la FNARC pour leur aimable et précieuse collaboration.

\section{BIBLIO GRAPHIE}

1. ALZIEU J.P., BICHET H., 1989. Réflexions sur la grippe équine et la rhinopneumonie équine. Moyens de contrôle. Revue Méd. vét., 140 : 1097-1107

2. CHABCHOUB A., GHRAM A., LOUZIR H., 1994. Maladies respiratoires du cheval : situation actuelle et incidence sur l'élevage équin en Tunisie. In : Journées sur les acquis de la recherche agronomique et vétérinaire, Hammamet, Tunisie, 2-4 décembre 1994.

3. CRU CIERE C., GUILLEM IN M.C., RO SETTO A., WIRBEL A., PLATEAU E., 1989. Production of monoclonal antibodies against influenza. Applications to a comparative study of various strains of virus. Ann. Rech. vét., 20: 243-250.

4. DELANNOY I., DUBOURGET P., FAYET G., 1995. Le point sur la rhinopneuomnie. Prat. vét. équine, 27 : 31-47.

5. ELLOUZE M.R., 1980. Contribution à l'étude épidémiologique de l'anémie infectieuse, de l'artérite à virus, de la rhinopneumonie et de la grippe équine en Tunisie. Thèse doct. vét., EN MV, Sidi-Thabet, Tunisie.

6. GHRAM A., CHABCHOUB A., TURKI I., BOUSSETTA M., IBN AMOR H., GHORBEL A., 1994. Rhinopneuomnie et artérite à virus des équidés : Enquête épidémiologique dans le Nord-Est de la Tunisie. Arch. Inst. Pasteur Tunis, $71:$ 5-12.

7. IBN AM OR H., 1993. Contribution à l'étude séro-épidémiologique de la rhinopneumonie et de l'artérite à virus en Tunisie. Thèse doct. vét., EN MV, Sidi-Thabet, Tunisie.

8. MERAI C., 1985. Contribution à l'étude de la rhinopneumonie équine en Tunisie. Situation dans les haras de Sidi-Thabet. Thèse doct. vét. EN MV, Sidi-Thabet, Tunisie.

9. MORAILLON A., MORAILLON R., TOMA B., SEDRATI A., LAHLOU KASSI S., 1978. Enquête épidémiologique de l'anémie infectieuse, de l'artérite à virus, de la rhinopneumonie et de la grippe équine au $\mathrm{M}$ aroc. Rec. M éd. vét., 154 : 921-928. 
10. PLATEAU E., JACQUET A., 1989. La rhinopneumonie équine situation épidémiologique en France, diagnostic et prévention. Prat. vét. équine, $4: 5-11$.

11. TOUMI M., 1985. Enquête sérologique sur la rhinopneumonie équine dans les haras nationaux en Tunisie. Thèse doct. vét., ENMV, Sidi-Thabet, Tunisie.

\section{Summary}

Ghram A., Chabchoub A., Boussetta M., Baazaoui S., Ibn Amor H., Landolsi F. A seroepidemiological survey on equine rhinopneumonitis in Tunisia

Specific antibodies to the equine rhinopneumonitis virus were detected using the complement fixation test in a seroepidemiological survey carried out on 789 equines (400 were bred in the North-East of Tunisia and 389 in the Sahel and the Centre). The results showed that 15 equines (1.9\%) were seropositive with various levels of complement fixation antibodies. These results are discussed in relation with those from other authors in Tunisia and neighboring countries.

Key words: Arabian horse - Thoroughbred horse - Barb horse Arab-Barb horse - Equine rhinopneumonitis virus Complement fixation test - Immunology - Epidemiology Tunisia.
12. VULCANO G. CHEYROUX M. JACQUET A. 1988. Rhinopneumonie équine à herpès virus equin-1. Comparaison des techniques de séro-neutralisation, de fixation du complément et d'ELISA. Rec. Méd. vét., 164 : 641-646.

13. ZIENTRA S., PLATEAU E., 1993. Vaccin et vaccination chez le cheval. Point vét., 24 : 601-610.

Reçu le 11.9.97, accepté le 7.1.98

\section{Resumen}

Ghram A., Chabchoub A., Boussetta M., Baazaoui S., Ibn Amor H., Landolsi F. Encuesta seroepidemiológica de la rinoneumonía de los equinos en Túnez

Se llevó a cabo una encuesta seroepidemiológica, en 789 équidos, de los cuales 400 criados en el Noreste de Túnez y 389 en la región del Sahel y del Centro, lo cual permitió detectar, mediante el test de fijación de complemento, los anticuerpos específicos contra el virus de la rinoneumonía equina. Los resultados muestran que 15 equinos $(1,9 \%)$, fueron seropositivos, con tasas variables de anticuerpos fijando el complemento. Estos resultados se discuten en relación con los obtenidos por otros autores en Túnez y en los países vecinos.

Palabras clave: Caballo de Pura sangre Arábigo - Caballo de Pura sangre Inglés - Caballo Barbe - Caballo Arábigo-Barbe Virus de la rinoneumonitis equina - Prueba de fijación del complemento - Inmunología - Epidemiología - Túnez. 